\title{
A novel engineered interferon- $\alpha$ hybrid molecule increases anticancer efficacy of doxorubicin in breast cancer chemotherapy
}

\author{
SI-JIE LI ${ }^{1}$, CHUN-SHUI LIU ${ }^{2}$, HAI-JUN LI ${ }^{3}$, YAN LI $^{2}$, LEI ZHOU $^{2}$, \\ JIN-CHENG $\mathrm{LI}^{4}$, YI-CHANG CHEN ${ }^{4}$, TIAN-QI SU ${ }^{4}$ and DE-HAI YU ${ }^{2}$ \\ ${ }^{1}$ Department of Breast Surgery, the First Hospital of Jilin University; ${ }^{2}$ Cancer Center, the First Hospital of \\ Jilin University; ${ }^{3}$ Institute of Translational Medicine, the First Hospital of Jilin University; ${ }^{4}$ Department of Clinical \\ Medicine, Norman Bethune Health Science Center, Jilin University, Changchun, Jilin 130021, P.R. China
}

Received April 17, 2017; Accepted June 19, 2017

DOI: $10.3892 /$ ijo.2017.4062

\begin{abstract}
Breast cancer is the most common carcinoma among Chinese women. Interferon $\alpha$ (IFN $\alpha$ ) has been used to treat various types of cancer, including breast cancer, but its antitumor activity is relative low, which significantly hinders its clinical application. In this study, we utilized a Ph.D.-12 peptide library screening system to identify a short peptide that specifically binds to MCF-7 breast cancer cells. By fusing the MCF-7 binding peptide (MBP) to the C-terminus of IFN $\alpha$, we constructed an engineered IFN $\alpha$-MBP fusion molecule (IMBP), and applied this novel fusion protein to the treatment of breast cancer. We found that IMBP exhibited significantly higher activity than wild-type IFN $\alpha$ in inhibiting cell growth and inducing cell apoptosis. Additionally, IMBP potentiated the therapeutic efficacy of doxorubicin-based breast cancer chemotherapy via the activation of cell cycle arrest and cell apoptosis pathway genes including p53, p21, CDK2, cyclin A, caspase 9, Bcl-2 and Bax. The enhanced activity of the synthetic IMBP was also associated with the activation of signal transducer and activation of transcription 1 (STAT1) pathway target genes (STAT1, IFIT1, IFITM1 and MX1). This study evaluated the potential value of the synthetic IMBP as a novel anti-breast cancer agent.
\end{abstract}

\section{Introduction}

In recent years, cytokine research has been at the forefront of cancer research and cytokine approaches are involved in the treatment of various carcinomas. Cytokine approaches for cancer therapy have three potential mechanisms of action. They can i) directly induce cell death programs in tumor cells,

Correspondence to: Dr De-Hai Yu, Cancer Center, the First Hospital of Jilin University, 71 Xinmin Street, Changchun, Jilin 130021, P.R. China

E-mail: yudehai@jlu.edu.cn

Key words: phage display library, interferon $\alpha$, doxorubicin, breast cancer ii) increase the number or activity of immune effector cells, or iii) increase the recognition of tumor cells by the immune system (1).

Interferons (IFNs) are one of the most important cytokines. They are naturally secreted glycoproteins produced by almost every cell type as a mechanism of host defense in response to microbial attack (2). The IFN family includes three different groups. IFN $\alpha$ belongs to the type I IFN group, and was discovered 50 years ago. It was the first cytokine to be produced by recombinant DNA technology, and it is used as an important regulator of cell growth and differentiation, affecting cellular communication and signal transduction pathways as well as immunological control (3). More recently, IFN $\alpha$ has been applied in the treatment of multiple carcinomas including leukemia, hepatocellular carcinoma, bladder cancer, and osteosarcoma (4-7).

Breast cancer is a leading cause of cancer-related death in women worldwide. According to GLOBOCAN 2012, there are approximately 1.67 million newly diagnosed breast cancer patients every year (8). In China, breast cancer is the most commonly diagnosed cancer, and the number of breast cancer patients has been increasing annually (9). Surgery, chemotherapy, radiotherapy, endocrinotherapy and molecular targeting therapy are the major treatment modalities for breast cancer. However, challenges remain in the treatment of breast cancer, and novel therapeutic approaches are urgently needed.

Although the use of IFN in clinical practice is widely recommended, its antineoplastic activity and clinical efficacy for breast cancer is still unclear and controversial $(10,11)$. In this study, an NEB Ph.D.-12 peptide library was employed to select a short peptide that specifically binds to the cell membrane of MCF-7 cells, and a high-affinity IFN $\alpha$-MCF-7 fusion molecule IMBP was constructed. Our aim was to investigate whether this reconstructed cytokine had enhanced cell growth inhibition activity compared to the wild-type IFN $\alpha$.

\section{Materials and methods}

Cell culture. Human breast cancer cell line MCF-7, lung cancer cell line A549 and prostate cancer cell line PC-3 were kept in our lab. Human embryonic kidney $293 \mathrm{~T}$ cell line was used for lentiviral packaging. All cell lines were routinely cultivated 
in Dulbecco's modified Eagle's medium (DMEM, Invitrogen, Carlsbad, CA, USA) with 10\% fetal bovine serum (Invitrogen), $100 \mathrm{U} / \mathrm{ml}$ penicillin and $100 \mu \mathrm{g} / \mathrm{ml}$ streptomycin (Invitrogen) at $37^{\circ} \mathrm{C}$ in a humidified atmosphere containing $5 \% \mathrm{CO}_{2}$.

Phage display library screening of breast cancer binding peptides. Phage display library (Ph.D.-12 library, \#E8110S, New England BioLabs, Ipswich, MA, USA) was employed to screen a short 12-peptide that specifically binds to MCF-7 breast cancer cells $(12,13)$. Briefly, $1 \times 10^{11}$ phage in $1 \mathrm{ml}$ DMEM medium was incubated with $1 \times 10^{6}$ MCF-7 cells at room temperature (RT) for $1 \mathrm{~h}$ to develop phage-cell complexes. After binding, cells were washed 5 times with $5 \mathrm{ml}$ TBST [TBS $(10 \mathrm{mmol} / \mathrm{l}$ Tris $\mathrm{pH} 7.5,150 \mathrm{mmol} / \mathrm{l} \mathrm{NaCl})$ containing $0.1 \%$ Tween-20] to remove the unbound phages. Cells were collected by centrifuging at $3200 \mathrm{x} \mathrm{g}$. The surface-bound phages were eluted in $1 \mathrm{ml}$ elution buffer [0.2 N Glycine-HCl (pH 2.2), $1 \mathrm{mg} / \mathrm{ml} \mathrm{BSA]} \mathrm{for} 20 \mathrm{~min}$ and was neutralized by $150 \mu 11 \mathrm{~N}$ Tris-HCl, $\mathrm{pH} 9.0$. The eluted phages were reproduced by infecting E. coli ER2738 and purified using polyethylene glycol (PEG)-8000/NaCl solution (20\% PEG-8000 and $2.5 \mathrm{~N}$ $\mathrm{NaCl})$.

After 4 rounds of surface panning, the DNA sequences of MCF-7-binding peptide (MBP) were amplified by PCR (Fig. 1A). Primers used for PCR amplification were as follows: 5'-CTTTAGTGGTACCTTTCTATTCTCGAGTCT-3' (forward primer with Xho $I$ ) and 5'-CTTTCAACAGTTTCGTCTAGA ACCTCCACC-3' (reverse primer with $X b a \mathrm{I}$ ). The PCR production was purified from $3 \%$ agrose gel and cloned into pJet vector using CloneJET PCR Cloning kit (Thermo Fisher Scientific, Waltham, MA, USA) for sequencing and constructing the engineered IFN $\alpha$ molecules.

Phage-ELISA assay. Phage-ELISA was carried out to determine the binding affinity of the isolated MBP. Approximately $2 \times 10^{4} \mathrm{MCF}-7$ cells were collected into a 96-well V-bottom tissue culture plate and incubated with blocking buffer (5\% BSA/PBS, Sigma, Shanghai, China) at $37^{\circ} \mathrm{C}$ for $1 \mathrm{~h}$. For comparison, A549 lung cancer and PC-3 prostate cancer cells were used as negative controls. Cells were pelleted by centrifuging at 3,200 $\mathrm{xg}$ and resuspended in $100 \mu \mathrm{l}$ PBS buffer (Thermo Fisher Scientific, Beijing, China) containing $1 \times 10^{9}$ phage particles. After incubated at RT for $1 \mathrm{~h}$, cells were pelleted and washed with PBST for $5 \times 5$ min. For quantitation, cells were incubated with $100 \mu \mathrm{l}$ PBS buffer containing HRP conjugated anti-M13 monoclonal antibody (1:5,000, GE Healthcare, Piscataway, NJ, USA) at $37^{\circ} \mathrm{C}$ for $45 \mathrm{~min}$. Cells were centrifuged and washed by PBST for $5 \times 5 \mathrm{~min}$. Then $100 \mu 1$ of TMB solution (BD Biosciences, San Jose, CA, USA) was added to each well and the plate was kept in the dark for 15 min until blue color was developed. Reaction was stopped by adding $50 \mu 11 \mathrm{~N}$ sulfuric acid. The absorbance of $450 \mathrm{~nm}$ and $630 \mathrm{~nm}$ were measured by microplate reader (Bioteck, Beijing, China). Final optical density (OD) was designated as $\mathrm{OD}_{450}-\mathrm{OD}_{630}-\mathrm{OD}_{\text {blank }}$.

Recombinant plasmid construction. After the phage library screening and sequencing, one of the MBP sequence was selected for the following cell studies. We ligated MBP to the C-terminus of IFN $\alpha$ to construct an engineered synthetic IFN $\alpha$ fusion protein. The fusion protein was generated by ligating the MBP sequence into IFN $\alpha$ vector at the $X h o I / X b a I$ restriction sites and was designated as IMBP. The wild-type IFN $\alpha$ expression plasmid was prepared as previous described $(14,15)$ and kept in our laboratory. The vector containing the isolated MBP sequence and the empty lentivirus vector were constructed in parallel and used as control groups. All plasmid constructs were confirmed by DNA sequencing.

The putative structure of the IMBP. The putative structure of the synthetic IMBP was predicted using online I-TASSER server (http://zhanglab.ccmb.med.umich.edu).

Lentivirus production. 293T cells were seeded into a 6-well plate at a proper density $24 \mathrm{~h}$ pre-transfection. Lentiviruses were packaged by the co-transfection of the plasmids constructed above and pSPAX2/pMD2.G packing vectors (System Biosciences, Mountain View, CA, USA) using Lipofectamine 3000 reagent (Thermo Fisher Scientific). The viral supernatants were collected at 24 and $48 \mathrm{~h}$ posttransfection and used for cell transfection as previously described $(16,17)$.

Infection of recombinant lentivirus. Approximately $2 \times 10^{5}$ MCF-7 cells were plated into a 6-well plate $24 \mathrm{~h}$ pre-transfection. Cells were infected with lentiviruses carrying MBP, IFN $\alpha$, IMBP and empty vector DNA in the presence of $8 \mu \mathrm{g} / \mathrm{ml}$ polybrene (Sigma). After two rounds of infection with lentiviruses at $37^{\circ} \mathrm{C}$ for $24 \mathrm{~h}$, the tissue culture medium was replaced with fresh complete medium. Three days after viral infection, the stable MCF-7 cells were selected by puromycin and used for the following cell experiments.

Detection of the secreted IMBP fusion protein. On day 5 post-transduction, $100 \mu 1$ supernatant of MCF-7 cells was collected and the secreted IFN $\alpha$ and IMBP fusion protein were quantitated with IFN $\alpha$ assay kit according to the manufacture's instruction (Cusabio, Hubei, China).

Western blotting. Protein were extracted with RIPA buffer (KeyGEN Biotech, Jiangsu, China) supplemented with cocktail protease inhibitor (Roche, Shanghai, China) and quantified with BCA protein assay kit (Beyotime Biotechnology, Jiangsu, China). Whole cell lysates were resolved on $5-10 \%$ or $5-12 \%$ SDS polyacrylamide gel electrophoresis (SDS-PAGE). To detect the secreted IFN $\alpha$ and IMBP proteins in cell supernatants, on day 5 post-transduction, $100 \mu 1 \mathrm{MCF}-7$ cell supernatant was collected and condensed to $20 \mu \mathrm{l}$ in a vacuum-freeze dryer (Boyikang, Beijing, China). Solutions were resolved on Mini-PROTEIN TGX gradient gel (Bio-Rad, Beijing, China). Proteins were transferred to $0.45 \mu \mathrm{m}$ PVDF membranes (Roche) and immunoblotted at $4^{\circ} \mathrm{C}$ overnight or at RT for $1 \mathrm{~h}$ with the following antibodies: IFN $\alpha$ (1:1,000, Abcam, Shanghai, China), STAT1 (phospho Y701, 1:1,000, Abcam), Cleaved CASP-9 (1:1,000, Cell Signaling Technology, Beijing, China), p53 (1:1,500, Santa Cruz Biotechnology, Santa Cruz, CA, USA), p21 (1:500, Santa Cruz Biotechnology), CDK2 (1:1500, Cell Signaling Technology), Cyclin A (1:1,500, Abcam) and $\beta$-actin (1:3,000, Santa Cruz Biotechnology). Membranes were incubated with horseradish peroxidase-conjugated secondary antibody 
(1:3,000, ZSGB-BIO, Beijing, China) at $37^{\circ} \mathrm{C}$ for $1 \mathrm{~h}$ before chemiluminescence reading. Protein expression levels were determined semi-quantitatively by densitometric analysis with the Quantity One software (Bio-Rad). Western blotting was performed in triplicate, and data showed a representative finding of these triplicate analyses.

Cell binding assay of IMBP. The binding affinity of the engineered hybrid molecule to the MCF-7 cell membrane was measured by FACS. MCF-7 cells were collected and stained with Trypan blue to make sure that viable cells were more than ninety percent. Approximately $1 \times 10^{6}$ normal MCF-7 cells were incubated with equal amount of the secreted IFN $\alpha$ or IMBP at $37^{\circ} \mathrm{C}$ for $1 \mathrm{~h}$. After washed with PBS, cells were incubated with FITC-conjugated IFN $\alpha$ antibody (PBL, Piscataway, NJ, USA). The FITC-conjugated mouse IgG (Abcam) was used as the isotype control. Cells were analyzed using BD LSRII Fortesa flow cytometer (BD Biosciences) and FlowJo software (FlowJo, OR, Ashland, USA) to calculate the fluorescence intensity $(14,15)$.

Cell viability assay. On day 7 post-transfection, approximately $5 \times 10^{3}$ viable stable MCF-7 cells transduced by lentivirus carrying IMBP, IFN $\alpha, \mathrm{MBP}$ and empty vector DNA were seeded into a 96 -well plate $24 \mathrm{~h}$ before cell viability assay. Then, cell growth was analyzed by WST-1 Cell Proliferation Reagent (Roche). According to the manufacturer's instructions, $20 \mu \mathrm{l}$ WST-1 reagent was added to $200 \mu \mathrm{l}$ cell culture medium and incubated at $37^{\circ} \mathrm{C}$ in the dark for $2 \mathrm{~h}$. The absorbances of 450 and $630 \mathrm{~nm}$ were measured with microplate reader (Bioteck). Final OD was designated as $\mathrm{OD}_{450}-\mathrm{OD}_{630}-\mathrm{OD}_{\text {blank }}$.

Cell cycle assay. On day 7 post-transfection, $1 \times 10^{6}$ stable MCF-7 cells were collected and washed by cold PBS. Cells were resuspended in $1 \mathrm{ml}$ fixation solution (300 $\mu \mathrm{l}$ PBS and $700 \mu \mathrm{l}$ ethanol). After incubation at $4^{\circ} \mathrm{C}$ for $4 \mathrm{~h}$, cells were centrifuged and the fixation solution was removed. After washed twice with PBS, cells were pelleted, stained with $0.5 \mathrm{ml}$ propidium iodide (PI, Sigma) staining solution [50 $\mu \mathrm{g} / \mathrm{ml} \mathrm{PI,} 20 \mu \mathrm{g} / \mathrm{ml}$ RNase A (Takara, Liaoning, China) and 0.2\% Triton X-100 (Sigma)], and incubated in the dark at $37^{\circ} \mathrm{C}$ for $10 \mathrm{~min}$. Cell suspensions were filtered with a 400-mesh sieve. Cell cycle was analyzed by BD LSRII Fortesa flow cytometer.

Flow cytometry for cell apoptosis. Annexin V-FITC and PI double staining flow cytometry analyses were used for cell apoptosis assay. Approximately $1 \times 10^{6}$ stable MCF-7 cells were collected and washed three times with cold PBS and binding buffer. Then cells were stained with Annexin V-FITC and PI (Annexin V-FITC Apoptosis Detection kit, BD Bioscience) for apoptosis detection. Briefly, MCF-7 cells were first resuspended in $1 \mathrm{ml}$ binding buffer. Then, $5 \mu \mathrm{l}$ of Annexin V-FITC was added to the tubes, and cells were incubated for $10 \mathrm{~min}$ at RT followed by the addition of $5 \mu \mathrm{l}$ PI. After 15 min incubation in PI buffer at RT, cells were immediately analyzed with a flow cytometer (BD Biosciences). The cells in the different portions represented the different cell states as follows: the late-apoptotic cells were present in the upper right portion, the viable cells were present in the lower left portion, and the early apoptotic were cells present in the lower right portion.
Treatment with chemotherapeutic drug doxorubicin. The anticancer drug doxorubicin (DOX, Sigma) was dissolved in water. We harvested $12 \mathrm{ml}$ of cell supernatant containing the secreted interferons from the stable MCF-7 cells transduced by lentivirus carrying IMBP, IFN $\alpha, \mathrm{MBP}$ and empty vector. The cell supernatant was centrifuged at 3,500 x $\mathrm{g}$ for $5 \mathrm{~min}$ to remove cell debris. Then the supernatant was successively passed through two centrifugal filter units with MW cut-off 30 and $10 \mathrm{kDa}$ (Millipore, Temecula, CA, USA) to remove large and small molecules. The interceptions were dissolved in $6 \mathrm{ml}$ DMEM complete medium and kept in $4^{\circ} \mathrm{C}$. Normal MCF-7 cells were seeded into 6-well plates at a density of $3 \times 10^{5}$ cells/well. After $24 \mathrm{~h}$, cell medium was changed to the interception-DMEM supplemented with $0.1 \mu \mathrm{g} / \mathrm{ml}$ DOX. MCF-7 cells were treated with this therapeutic medium for 3 days, with the medium changed every day. After that, cells were harvested and applied for the next step of cell viability assay or molecule detection.

Real-time PCR. Total cellular RNA was isolated using an Eastep Super total RNA isolation kit (Promega, Beijing, China). First-strand cDNA was synthesized from $800 \mathrm{ng}$ of total RNA using Transcriptor First Strand cDNA Synthesis kit (Roche). Real-time PCR was performed using an aliquot of first-strand cDNA as a template in a $20 \mu \mathrm{l}$ reaction system containing $10 \mu \mathrm{l} 2 \mathrm{X}$ SYBR premixed buffer (Roche), $2 \mu 1$ forward and reverse primers. The primers were as follows: IFIT1 (interferon induced protein with tetratricopeptide repeats) sense 5'-TCTCAGAGGAGCCTGGCTAA-3', IFIT1 antisense 5'-CCAGACTATCCTTGACCTGATGA-3'; MX1 (MX dynamin-like GTPase 1) sense: 5'-CTTTCCAGTCCAG CTCGGCA-3', MX1 antisense: 5'-AGCTGCTGGCCGT ACGTCTG-3'); STAT1 sense: 5'-GGCACCAGAACGAATG AGGG-3', STAT1 antisense: 5'-CCATCGTGCACATGGTG GAG-3' (18); IFITM1 (interferon-induced transmembrane protein 1) sense: 5'-ACAGGAAGATGGTTGGCGAC-3', IFITM1 antisense: 5'-ATGGTAGACTGTCACAGAGC-3'; $\beta$-actin sense: 5'-TCACCCACACTGTGCCCATCTACGA-3', $\beta$-actin antisense: 5'-CAGCGGAACCGCTCATTGCCAA TGG-3' (19). The PCR amplification process was one cycle at $95^{\circ} \mathrm{C} 10 \mathrm{~min}, 40$ cycles at $95^{\circ} \mathrm{C}$ for $10 \mathrm{sec}$ and $60^{\circ} \mathrm{C}$ for $30 \mathrm{sec}$ (ABI StepOnePlus, Beijing, China).

Statistical analysis. All data and results were calculated from at least three replicate measurements and presented as means \pm SD. The significance was determined by SPSS 20.0 (IBM). Student's t-test was used to compare statistical differences for variables among treatment groups. $\mathrm{P}<0.05$ was considered to indicate a statistically significant difference.

\section{Results}

Construction of the IFNa/MCF-7-binding peptide fusion protein. In order to enhance the antitumor activity of IFN $\alpha$ in breast cancer therapy, we used a NEB Ph.D.-12 peptide library to select a short peptide with 12 amino acids that specifically binds to the cell membrane of MCF-7 cells. Our aim is to add a short, high-affinity peptide to the C-terminus of IFN $\alpha$ and construct an IFN $\alpha-M C F-7$ fusion molecule (IMBP), which may facilitate the binding of IFN $\alpha$ to the 
A

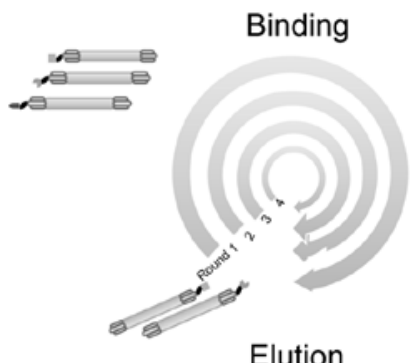

Elution
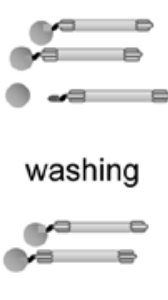

C

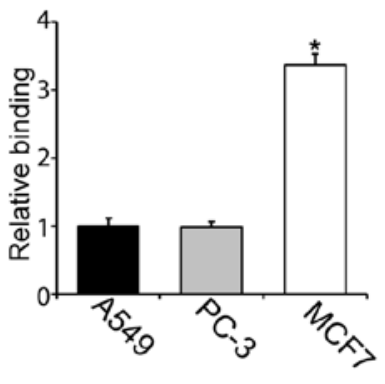

B

S CEHIKDELV C QNGGG

E

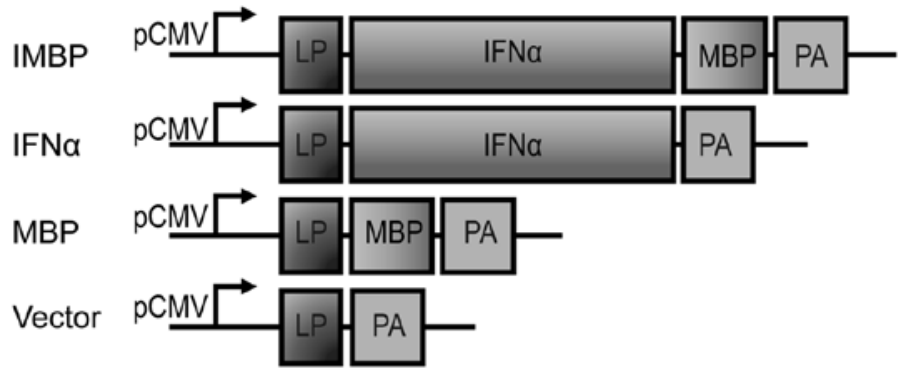

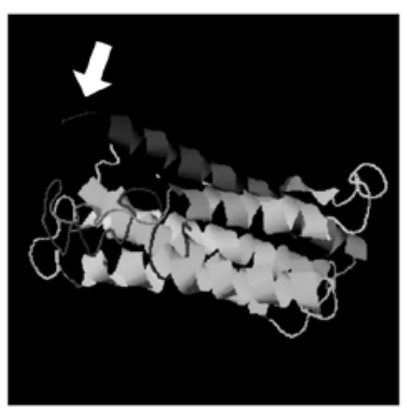

IFNa

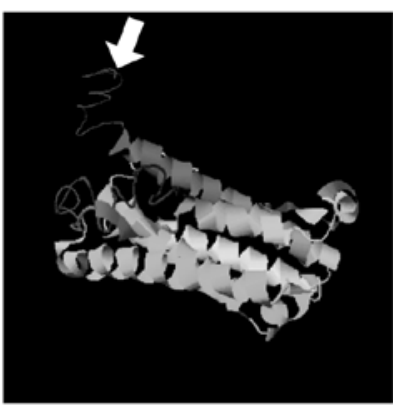

IMBP

Figure 1. Construction of the IFN $\alpha / \mathrm{MCF}-7-$ binding peptide fusion protein. (A) Ph.D.-12 peptide library screening of the MCF-7 breast cancer cell binding peptides. MCF-7 cells were incubated with phage display library and the cell-specific binding phages were screened following the manufacturer's protocol. After 4 rounds of surface panning, the DNA sequences encoding the MBPs were cloned and sequenced. (B) The amino acid sequence of MBP that was selected for the cell study. (C) Cell binding affinity assay of the screened MBP-carrying phage particles. MBP-phage particles were incubated with A549, PC-3 and MCF-7 cells. The binding affinity of the MBP-carrying phage was quantitated by phage-ELISA method. * P $<0.05$ as compared with A549 and PC-3 cells. (D) Construction of IMBP fusion protein expression vector. pCMV, CMV promoter; IFN $\alpha$, wild-type interferon $\alpha$; MBP, the control construct that contains only MCF-7 binding phage-display peptide sequence; IMBP, IFN $\alpha$ and MBP fusion protein expression vector; Vector, empty vector control. (E) The predicted protein structure of the synthetic IMBP. The putative structure of IMBP was predicted using software on the website: http://zhanglab.ccmb.med.umich.edu. Arrow indicates MBP fused into wild-type IFN .

MCF-7 cell membranes and enhance the cell growth inhibition activity of IFN $\alpha$.

According to the manual of NEB Ph.D.-12 peptide library, MCF-7 cells were incubated with $1 \times 10^{11}$ phage particles in DMEM tissue culture medium. The unbounded phage particles were stripped off by stripping buffer. The bound phages on the cell membrane were eluted and recovered for the second round of screening. After four rounds of screening, the phages specifically binding to MCF-7 cells were enriched and identified (Fig. 1A). After cloning and sequencing, one sort of phage encodes short peptide with the sequence of 'C E H I K D E L V C Q N' was selected for further cell studies (Fig. 1B). Using phage-ELISA, we showed that this phage displayed short peptide was able to bind preferentially to MCF-7 cells compared to A549 lung cancer and PC-3 prostate cancer cells (Fig. 1C).

In order to examine the role of this short peptide and compare the antitumor activity of wild-type IFN $\alpha$ and IMBP, we linked the short peptide to the C-terminus of IFN $\alpha$ and the IMBP fusion molecule was synthesized (Fig. 1D). Then we used software on the website (http://zhanglab.ccmb.med. umich.edu) to predict the protein structure of the synthetic IMBP. The structure of wild-type IFN $\alpha$ and the putative structure of IMBP is illustrated in Fig. 1E. Arrow indicates the short peptide fused into wild-type IFNa.

Detection of the secreted IFN $\alpha$ and IMBP fusion protein. We used 293T cells to produce lentivirus carring the DNA 
sequences of IMBP, wild-type IFN $\alpha$, MBP and empty vector. Then, MCF-7 cells were transducted by lentiviruses and the stable cells were selected with puromycin. On day 5 posttransduction, $100 \mu \mathrm{l}$ supernatant of MCF-7 cells was collected and the secreted IFN $\alpha$ and IMBP fusion protein were quantitated with IFN $\alpha$ assay kit. Based on the result of IFN $\alpha$ ELISA, the concentration of secreted wild-type IFN $\alpha$ in MCF-7 supernatant was $885 \mathrm{pg} / \mathrm{ml}$ and that of the IMPB fusion molecule was $763 \mathrm{pg} / \mathrm{ml}$ (Fig. 2A). We further used western blotting to confirm the secretion of wild-type IFN $\alpha$ and IMPB fusion molecule. The blots are shown in Fig. $2 \mathrm{~B}$.

Since the phage particles bind to MCF-7 cell membrane specifically, we used FACS to further examine whether the IMBP fusion protein binds to MCF-7 cell surface easier than the wild-type IFN $\alpha$. Therefore, FITC conjugated-IFN $\alpha$ antibody was used in FACS assay and the binding affinity of the two proteins was quantitatively evaluated according to the fluorescence intensity in flow cytometry (Fig. 2C). Quantitative analysis result showed that the binding affinity of the IMBP fusion protein was 1.5-fold increase compared to the wild-type IFN $\alpha(\mathrm{P}<0.05)$, and the binding ability of both the wild-type IFN $\alpha$ and the IMBP fusion protein was significantly higher than the isotype control (Fig. $2 \mathrm{C}, \mathrm{P}<0.05$ ).

Synthetic IMBP fusion protein inhibits cell growth of MCF-7 cells. We compared the cell growth inhibition effect between the IMBP and the wild-type IFN $\alpha$. MCF-7 breast cancer cells were transfected with lentiviruses carrying IMBP, IFN $\alpha$, MBP and empty vector, respectively. Cell proliferation was determined by WST-1 assay. We found that although both the secreted IFN $\alpha$ and IMBP inhibited the growth of MCF-7 cells, IMBP was superior to IFN $\alpha$ (Fig. 3A, $\mathrm{P}<0.05$ ).

Then we examined cell apoptosis of the stable transfected MCF-7 cells with flow cytometry. As was shown in Fig. 3B, we found that the transfection of both IFN $\alpha$ and IMBP induced an increased apoptosis ratio, but the IMBP group showed a significantly higher apoptosis ratio than the IFN $\alpha$ group (26 vs. 18\%, $\mathrm{P}<0.05$ ). The cell morphology also showed a similar result (Fig. 3C).

IMBP potentiates the therapeutic efficacy of doxorubicinbased chemotherapy. Since doxorubicin is one of widely used anthracyclines to treat breast cancer, we examined the synergistic effect on cell killing by the combined use of DOX and IMBP fusion molecule. First, using WST-1 cell proliferation assay, we tested a serial working concentration of DOX including $0.02,0.05,0.1,0.2,0.5$ and $1.0 \mu \mathrm{g} / \mathrm{ml}$ for the cell growth inhibition of MCF-7 cells. We selected a relative low concentration of $0.1 \mu \mathrm{g} / \mathrm{ml}$ of DOX with a cell growth inhibition ratio approximately $25 \%$ for the chemotherapy (data not shown). Then, we investigated the cell killing ability of the co-administration of DOX/IFN $\alpha$ or DOX/IMBP. The WST-1 assay result showed that cell proliferation in both the DOX/ IFN $\alpha$ and DOX/IMBP treatment groups was apparently inhibited compared to the PBS control group and the DOX group. Of note, cell killing ability of the co-administration of DOX and IMBP was superior to the co-administration of DOX and IFN $\alpha$ (Fig. 4A, 48 vs. 32\%, P<0.05).

Then we examined the cell cycle distribution in the treated MCF-7 cells with flow cytometry. We found that the
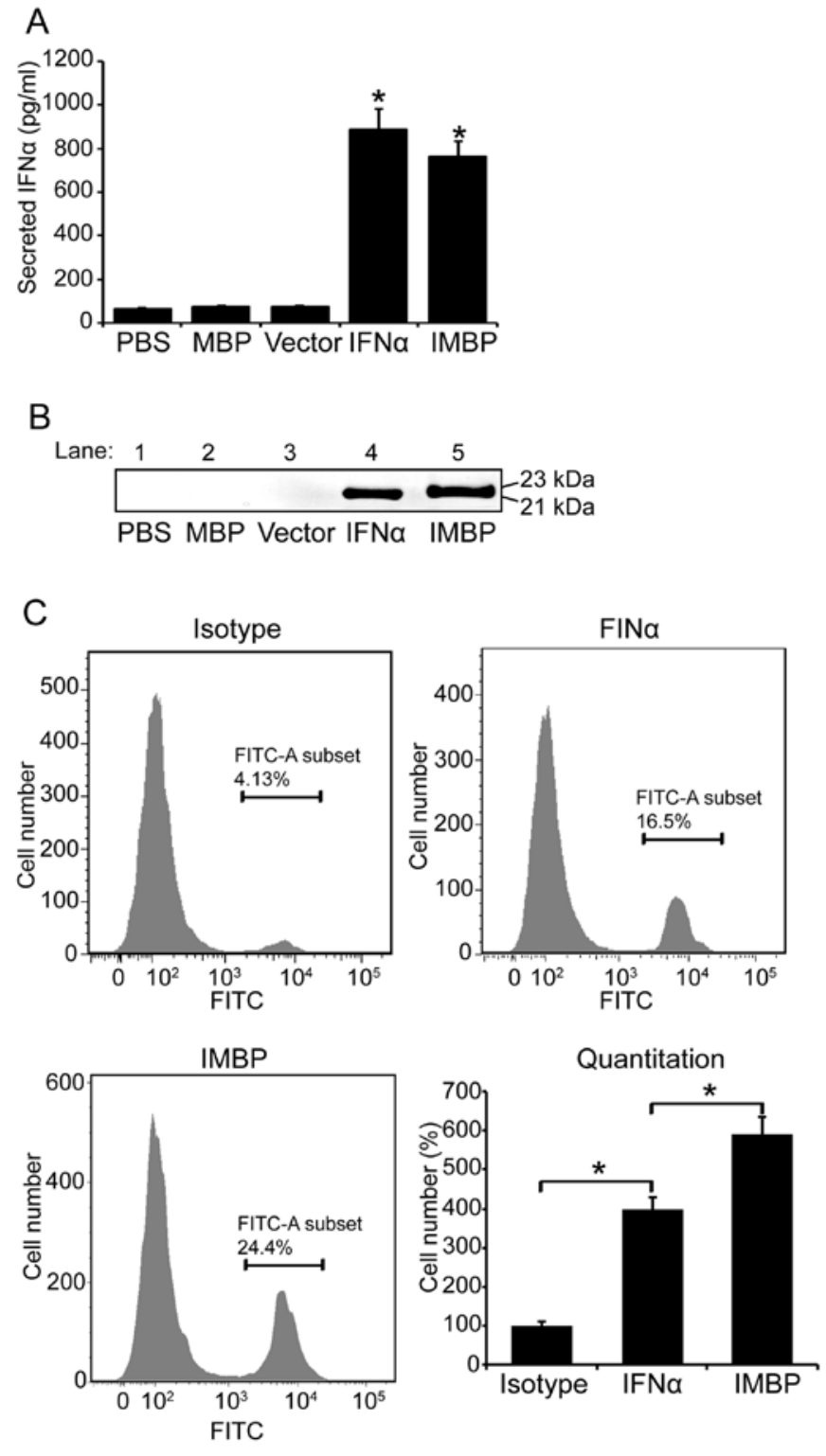

Figure 2. Detection of the secreted IFN $\alpha$ and IMBP fusion protein. (A) Detection of the secreted IFN $\alpha$ and IMBP fusion protein with IFN $\alpha$ ELISA kit. 293T cells were transfected with IMBP, wild-type IFN $\alpha$, MBP DNA sequence and empty vector. At 24 and $48 \mathrm{~h}$ post-transfection, cell supernatant containing lentiviruses was harvested. MCF-7 cells were transducted by lentiviruses and stable cells were selected with puromycin. At day 5 post-transduction, $100 \mu \mathrm{l}$ MCF-7 cell supernatant was collected and the secreted IFN $\alpha$ and IMBP fusion protein were quantitated with IFN $\alpha$ assay kit. (B) Detection of the secreted IFN $\alpha$ and IMBP fusion protein with western blotting. At day 5 post-transduction of MCF-7 cells, $100 \mu 1$ cell supernatant was collected and condensed to $20 \mu \mathrm{l}$ in a vacuum-freeze dryer. Then the cell supernatant was resolved by Mini-PROTEIN TGX gradient gel for western blot analysis. IMBP: $23 \mathrm{kDa}$, IFN $\alpha$ : $21 \mathrm{kDa}$ as estimated by ExPASy (http://web.expasy.org/cgi-bin/compute_pi/pi_tool). (C) Analyses of the cell membrane binding of IFN $\alpha$ and IMBP with FACS. Approximately $1 \times 10^{6}$ MCF-7 cells were inchubated with stable cell supernatant containing equal amount of secreted IFN $\alpha$ and IMBP. The FITC conjugated-IFN $\alpha$ antibody was used for FACS quantitation of the cell binding ability assay. The FITC conjugated mouse IgG was used as the isotype control. The fluorescence intensity was measured to evaluate the cell binding ability.

co-administration apparently induced an $\mathrm{S}$ phase cell cycle arrest, and the DOX/IMBP treatment group showed a more obvious arrest effect than the DOX/IFN $\alpha$ group (Fig. 4B, 43 vs. $37 \%, \mathrm{P}<0.05$ ). 
A

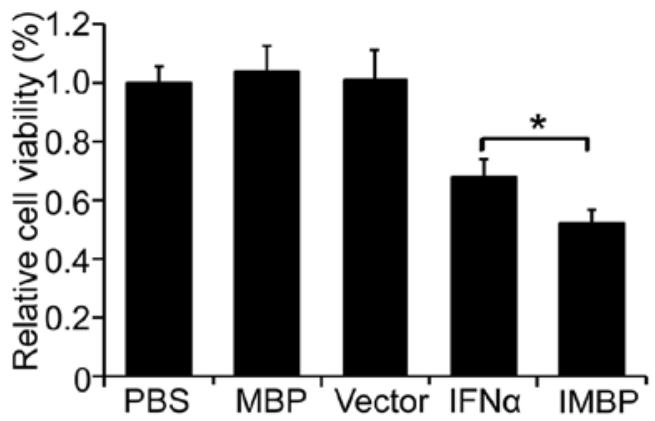

B
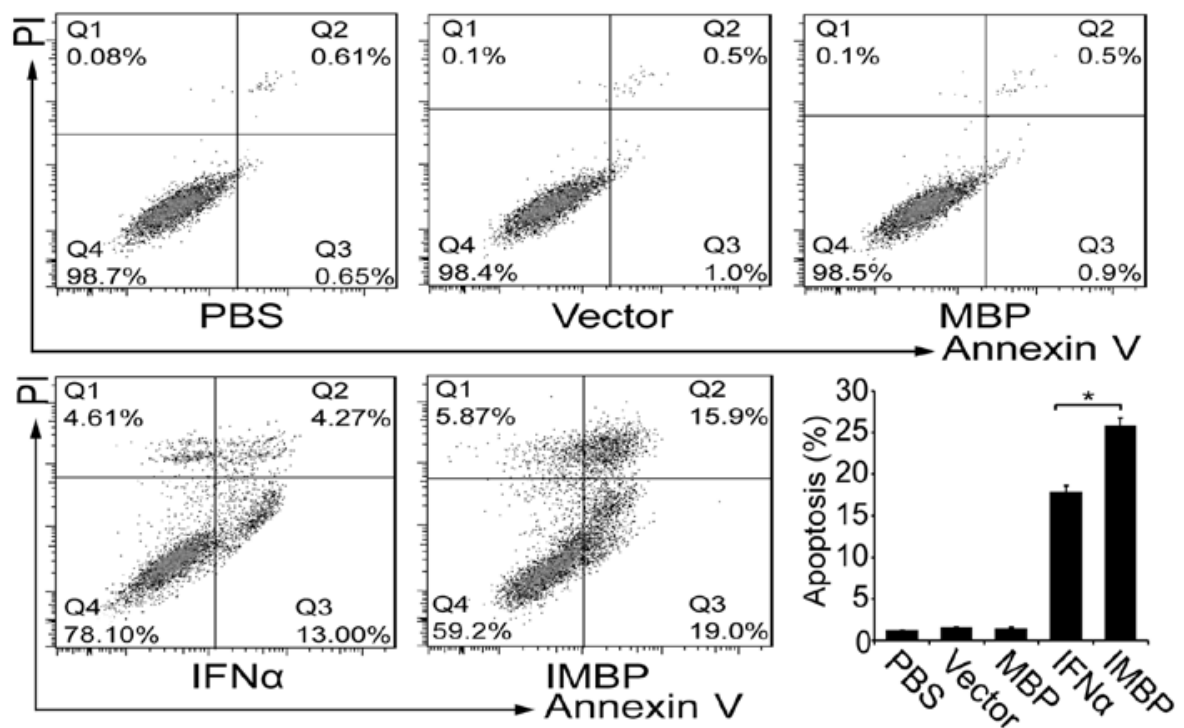

C
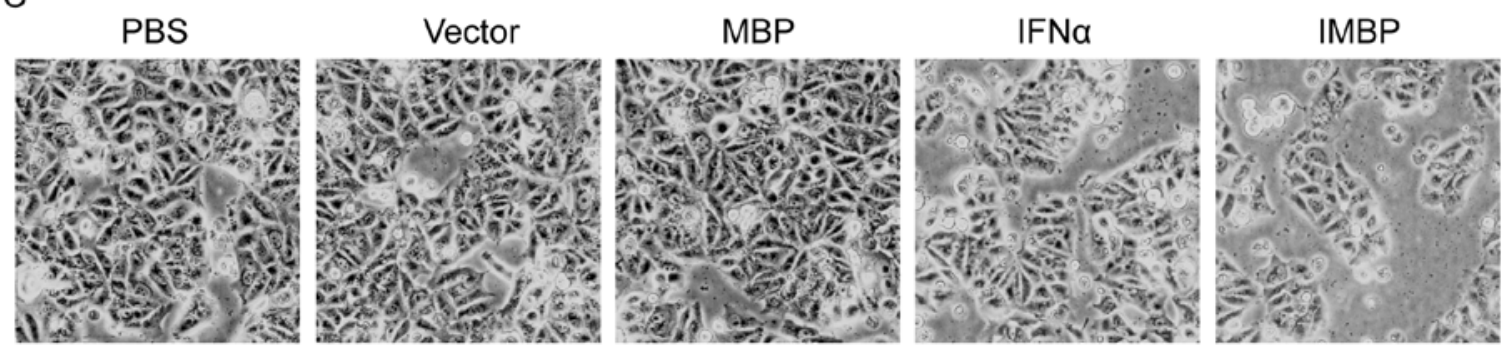

Figure 3. The synthetic IMBP fusion protein inhibits cell growth of MCF-7 breast cancer cells. (A) Cell viability of MCF-7 breast cancer cells. MCF-7 cells were transduced by lentiviruses carrying IMBP and IFN $\alpha$, MBP and empty vector sequences, and the stable cells were selected by puromycin. On day 7 post-transduction, approximately $5 \times 10^{3}$ viable stable MCF-7 cells were seeded in 96 -well and cell viability was determined by WST-1 method. (B) Cell apoptosis of the stable MCF-7 breast cancer cells were analyzed on day 7 after viral transduction by flow cytometry. Cell morphology were recorded on day 7 after viral transduction. " $\mathrm{P}<0.05$ as compared with the IFN $\alpha$ group.

To unveil the underlying molecular mechanism that explains the enhanced therapeutic efficacy of the combined use of DOX and IMBP in chemotherapy, we first examined the expression of the cell cycle pathway related genes by western blotting. We found that the expression of p53 and p21 in the DOX/IMBP chemotherapy group was dramatically up-regulated compared to the DOX/IFN $\alpha$ group; the expression of CDK2 and cyclin A was dramatically down regulated (Fig. 4C left panel and D, $\mathrm{P}<0.05)$. We then examined the expression of the cell apoptosis pathway-related genes and found that the caspase 9 and
Bcl-2/Bax apoptosis pathways were activated (Fig. 4C right panel and $\mathrm{D}, \mathrm{P}<0.05)$.

IMBP fusion molecule activates the STAT1 pathway. To further delineate the mechanism of the enhanced activity of the combination chemotherapy, we used real-time PCR to examine the expression of the interferon pathway genes in the treated MCF-7 cells. The quantitation result showed that both the DOX/IMBP and DOX/IFN $\alpha$ co-administration activate several inducible genes of the STAT1 pathway, including 
A

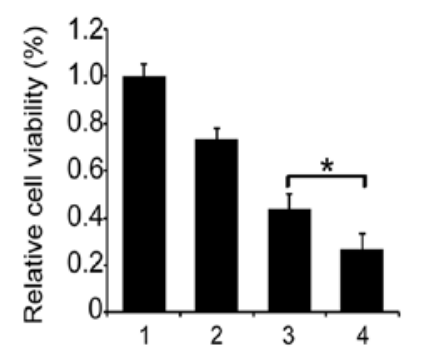

C

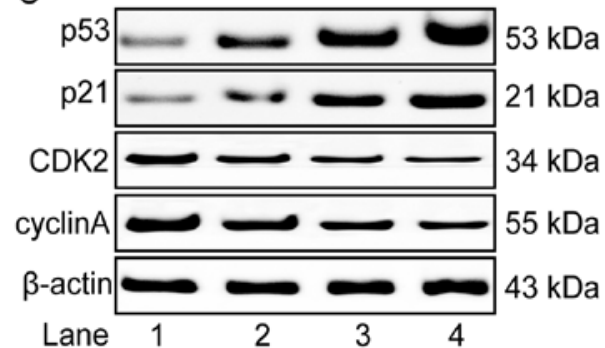

B

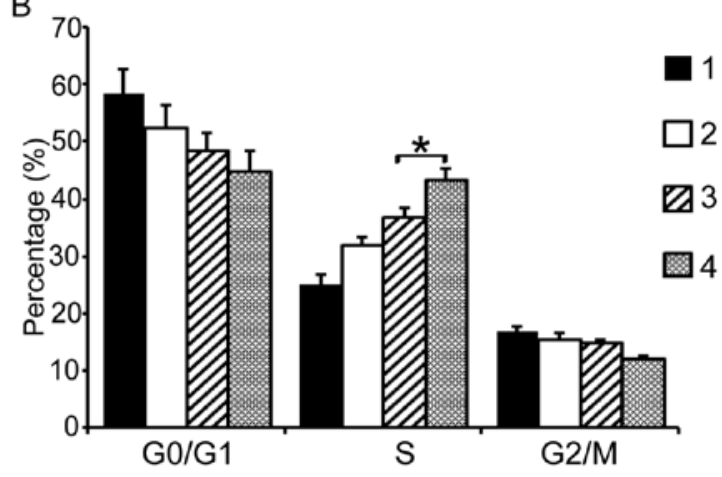

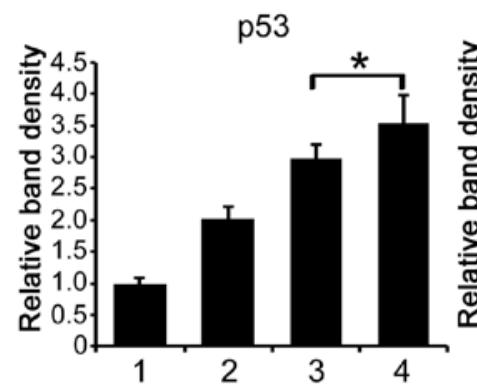
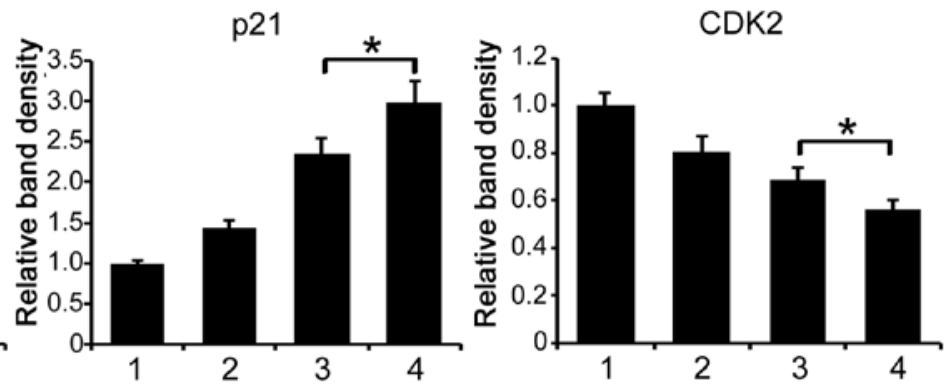

cyclin A

Cleaved Casp-9
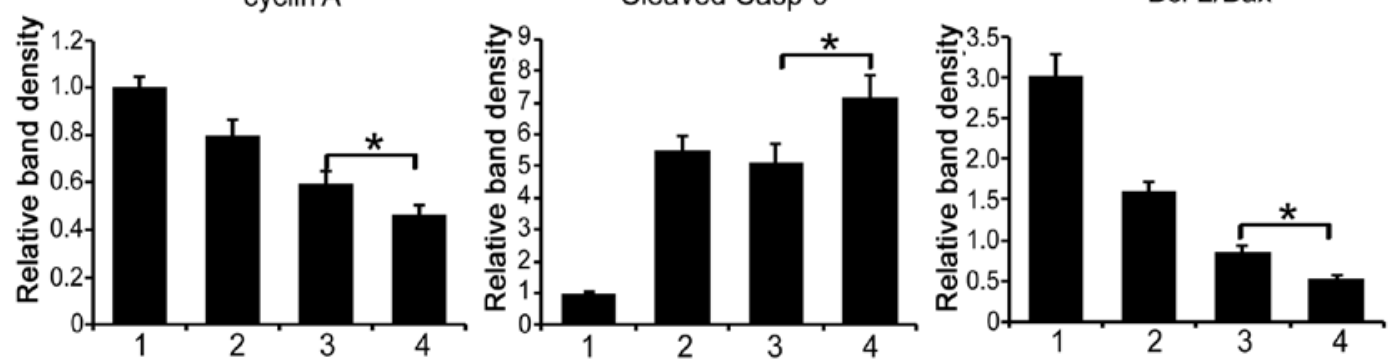

Figure 4. IMBP potentiates the therapeutic effect of doxorubicin-based chemotherapy. (A) MCF-7 breast cancer cells were treated with chemotherapeutic medium for 3 days and cell growth was measured by WST-1 assay. (B) Cell cycle distrubution of the treated MCF-7 breast cancer cells was analyzed by flow cytometry. (C) Western blot analyses of cell cycle and apoptosis related genes. (D) Relative band density of western blotting. 1: PBS control group; 2: DOX group; 3: IFN $\alpha$ plus DOX group; 4: IMBP plus DOX group. * $\mathrm{P}<0.05$ as compared with the IFN $\alpha$ plus DOX group.

STAT1, IFIT1, IFITM1, and MX1. However, DOX/IMBP group showed a stronger activation effect than the DOX/IFN $\alpha$ group (Fig. 5A). The western blot analysis result also showed an activation of the phosphorylated STAT1 (Y701, Fig. 5B).

\section{Discussion}

Anthracyclines, one type of anticancer therapy, are commonly used to treat both early and metastatic breast cancer; however, their toxicity, especially cardiac toxicity, is high $(20,21)$.
Additionally, frequent use of single therapeutic agent may result in chemoresistance, which is a major obstacle to the successful treatment of breast cancer. To minimize the toxicity of DOX and maximize the therapeutic efficiency of DOX, a combination therapeutic strategy is important.

The functions of IFNs are represented by three major biological activities, antiviral activity, antitumor activity and immunoregulatory activity (22). Although IFN $\alpha$ is widely used in the treatment of various types of cancer, its antineoplastic potency for breast cancer is low. Therefore, 

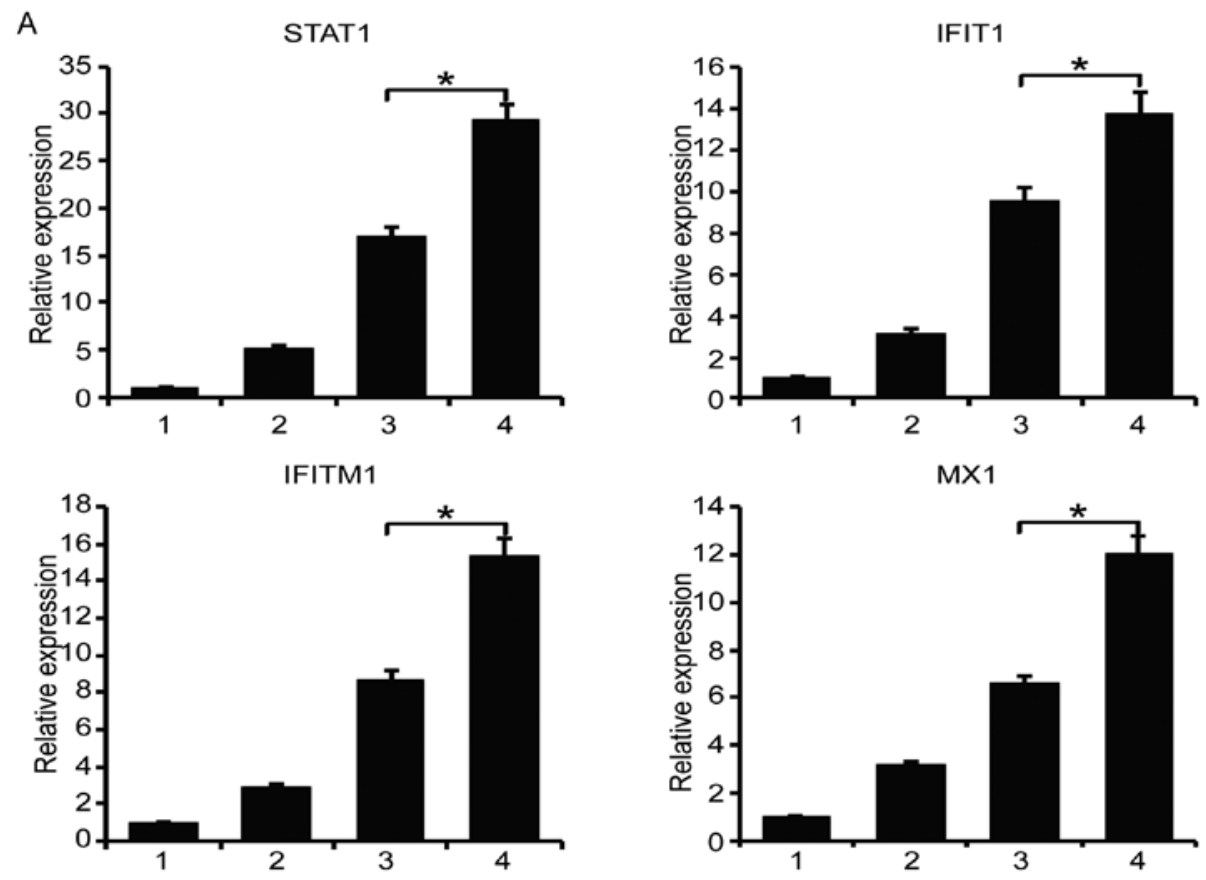

B
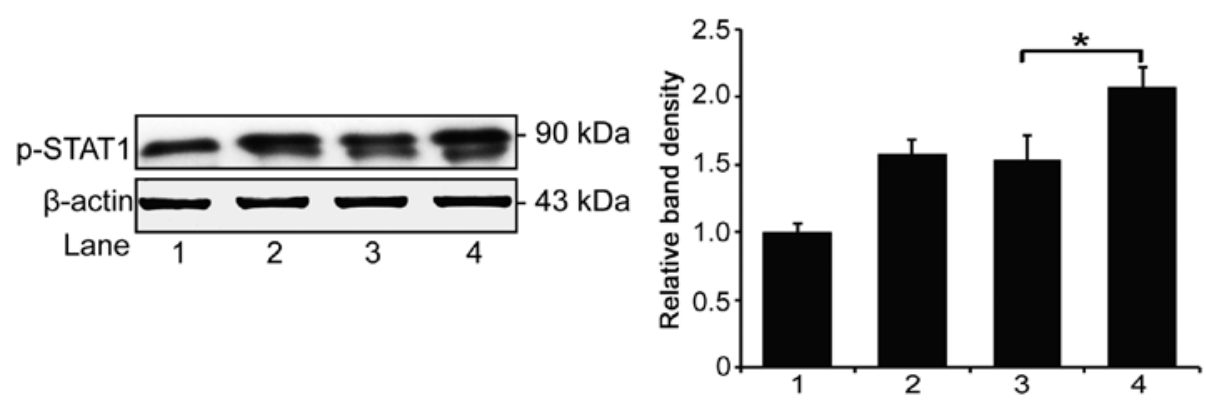

Figure 5. IMBP activates the STAT1 signal pathway in MCF-7 chemotherapy. (A) Real-time PCR analyses of the activation of the STAT1 signal pathway. (B) Western blot analyses of the STAT1 expression and the relative band density of western blots. 1: PBS control group; 2: DOX group; 3: IFN $\alpha$ plus DOX group; 4: IMBP plus DOX group. ${ }^{*} \mathrm{P}<0.05$ as compared with the IFN $\alpha$ plus DOX group.

improvements in this cytokine are valuable and further investigation is still needed.

In our previous study, using a cDNA in-frame library screening approach, we identified a short peptide derived from placental growth factor-2 (PLGF-2). We demonstrated that fusing this short peptide to IFN $\alpha$ and IFN $\gamma$ induced greater activity than the wild-type counterparts in inhibiting of tumor cell growth, invasion and colony formation $(14,15)$. In this study, using a Ph.D.-12 peptide library, we selected a short peptide that specifically binds to the cell membrane of MCF-7 cells, and we synthesized a high-affinity IFN $\alpha$-MCF-7 fusion molecule (IMBP). Using lentiviral DNA delivery system, we obtained a stable MCF-7 breast cancer cell line that secretes IMBP. Compared with wild-type IFN $\alpha$, the IMBP fusion molecule demonstrated higher antitumor activity potency in the inhibition of cell growth, promotion of cell cycle arrest and induction of cell apoptosis. Recently, using the same approach, we also identified a short peptide that specifically binds to Jurkat T lymphocyte leukemia cells (JBP). We demonstrated that a JBP and IFN $\alpha$ fusion protein (IFNP) also had significantly better antitumor activity than wild-type IFN $\alpha$ (unpublished data). Through this series study, we evaluated the value of engineering an IFN $\alpha$ fusion molecule, which may serve as a novel antitumor agent in cancer treatment.

IFNs bind to receptors on the cell surface, activate specific signaling pathways and exert antitumor actions (23). Unfortunately, we still do not know the function of how this Ph.D.-12 peptide binds to the cell membrane. According to the result of 'Blastp' on the NCBI website, this 12-peptide is not homologous to any existing protein sequences. We speculate that it may bind to some unknown receptor(s) or biomacromolecule(s) on the surface of the cell membrane. Using a cell binding assay, we demonstrated that MBP promoted the adherence affinity of IFN $\alpha$ to its receptor (Fig. 2C), which is probably why we detected a lower level of free IMBP in the cell supernatant (Fig. 2A). We speculate that because more IMBP molecules adhere to the cell surface of MCF-7 cells than IFN $\alpha$, the IMBP possesses a higher tumor cell killing ability than wild-type IFN $\alpha$.

The STAT pathway plays a critical role in the anti-infection role of IFNs (24-27). When binding to its receptor on the cell surface, IFN $\alpha$ initiates the phosphorylation of TYK2 and JAK1 kinases, which is followed by the activation of STAT family transcription factors $(28,29)$. Several IFN inducible 
genes can be co-activated in the STAT pathway, including IFIT1, IFIT3, MX1, IFITM1, OAS1 (2'-5'-oligoadenylate synthetase 1), PARP9 and PARP12 [poly(ADP-ribose) polymerase family] (30). It was reported that IFIT3 promotes IFN $\alpha$ adjuvant therapeutic effects by strengthening IFN $\alpha$ effector signaling in hepatocellular carcinoma patients (31); IFN $\gamma$ inhibits the growth of human liver cancer cells through activating IFITM1, which enhances the transcriptional activity of p53 and stabilizes the p53 protein by inhibiting p53 phosphorylation on Thr55 (19).

The JAK-STAT signaling pathway also plays an important role in doxorubicin-based breast cancer chemotherapy (32). It was demonstrated that the combined use of doxorubicin and IFNs may have a significant synergistic effect. Thomas et al showed that doxorubicin potentiates STAT1 activation in response to IFN $\gamma$, and the combination of doxorubicin and IFN $\gamma$ enhances apoptosis in breast cancer cells (33). Hannesdóttir et al reported that STAT1 is crucial to the susceptibility of breast cancer cells to chemotherapeutic drugs (e.g., doxorubicin) by contributing to the induction of a productive antitumor immune response, which is based on IFN $\gamma$-producing T cells (34). In this study, we found that a combination of low dose DOX antitumor drug $(0.1 \mu \mathrm{g} / \mathrm{ml})$ and endogenous secretory IFN $\alpha$ had an advantage over the single use of either DOX or IFN $\alpha$ in inhibiting breast cancer cell growth. Of note, we found that low dose DOX combined with synthetic IMBP fusion protein had a significantly better therapeutic effect than the DOX/IFNa combination. When we examined the molecular mechanism of this enhanced antitumor activity, we observed that the DOX/IMBP combination activated cell cycle arrest and cell apoptosis pathway genes more efficiently than the DOX/IFN $\alpha$ combination (Fig. 4), which was also correlated with the activation of STAT1 pathway target genes (Fig. 5).

In summary, by using a phage library screening, we have identified a short peptide that specifically binds to MCF-7 breast cancer cells. Fusion of this short peptide to IFN $\alpha$ significantly enhanced the antitumor activity. The combined use of DOX and IMBP fusion protein potentiated the effectiveness of chemotherapy. Since using lentivirus to deliver molecules into cells is hard to apply in clinical gene therapy, construction of an in vitro expression and purification system to produce purified IMBP recombinant protein is needed in our further study. We consider this type of purified IMBP molecule may have good clinical application prospect and the co-administration of DOX and IMBP provides new insight into breast cancer treatment.

\section{Acknowledgements}

The present study was supported by grants from the National Science Foundation of China (81302380, to D.-H.Y.) and Grant from the Health and Family Planning Commission in Jilin Province of China (2016Q035, to L.Z.).

\section{References}

1. Roychowdhury S and Caligiuri MA: Cytokine therapy for cancer: Antigen presentation. Cancer Treat Res 123: 249-266, 2005.

2. Kotredes KP and Gamero AM: Interferons as inducers of apoptosis in malignant cells. J Interferon Cytokine Res 33: 162-170, 2013.
3. Gutterman JU: Cytokine therapeutics: Lessons from interferon alpha. Proc Natl Acad Sci USA 91: 1198-1205, 1994.

4. Lamm D, Brausi M, O'Donnell MA and Witjes JA: Interferon alfa in the treatment paradigm for non-muscle-invasive bladder cancer. Urol Oncol 32: e21-e30, 2014.

5. Whelan J, Patterson D, Perisoglou M, Bielack S, Marina N, Smeland $S$ and Bernstein $M$ : The role of interferons in the treatment of osteosarcoma. Pediatr Blood Cancer 54: 350-354, 2010.

6. Wang L, Jia D, Duan F, Sun Z, Liu X, Zhou L, Sun L, Ren S, Ruan Y and Gu J: Combined anti-tumor effects of IFN- $\alpha$ and sorafenib on hepatocellular carcinoma in vitro and in vivo. Biochem Biophys Res Commun 422: 687-692, 2012.

7. Simonsson B, Hjorth-Hansen H, Bjerrum OW and Porkka K: Interferon alpha for treatment of chronic myeloid leukemia. Curr Drug Targets 12: 420-428, 2011.

8. Ferlay J, Soerjomataram I, Dikshit R, Eser S, Mathers C, Rebelo M, Parkin DM, Forman D and Bray F: Cancer incidence and mortality worldwide: Sources, methods and major patterns in GLOBOCAN 2012. Int J Cancer 136: E359-E386, 2015.

9. Fan L, Strasser-Weippl K, Li JJ, St Louis J, Finkelstein DM, Yu KD, Chen WQ, Shao ZM and Goss PE: Breast cancer in China. Lancet Oncol 15: e279-e289, 2014.

10. Ramos MC, Mardegan MC, Tirone NR, Michelin MA and Murta EF: The clinical use of type 1 interferon in gynecology. Eur J Gynaecol Oncol 31: 145-150, 2010.

11. Wang BX, Rahbar R and Fish EN: Interferon: Current status and future prospects in cancer therapy. J Interferon Cytokine Res 31: $545-552,2011$

12. Li X and Mao C: Using phage as a platform to select cancer celltargeting peptides. Methods Mol Biol 1108: 57-68, 2014.

13. Cao B, Yang M and Mao C: Phage as a genetically modifiable supramacromolecule in chemistry, materials and medicine. Acc Chem Res 49: 1111-1120, 2016.

14. Yin H, Chen N, Guo R, Wang H, Li W, Wang G, Cui J, Jin H and $\mathrm{Hu}$ JF: Antitumor potential of a synthetic interferon-alpha/ PLGF-2 positive charge peptide hybrid molecule in pancreatic cancer cells. Sci Rep 5: 16975, 2015.

15. Liu Y, Chen N, Yin H, Zhang L, Li W, Wang G, Cui J, Yang B and Hu JF: A placental growth factor-positively charged peptide potentiates the antitumor activity of interferon-gamma in human brain glioblastoma U87 cells. Am J Cancer Res 6: 214-225, 2016.

16. Sun J, Li W, Sun Y, Yu D, Wen X, Wang H, Cui J, Wang G, Hoffman AR and Hu JF: A novel antisense long noncoding RNA within the IGF1R gene locus is imprinted in hematopoietic malignancies. Nucleic Acids Res 42: 9588-9601, 2014.

17. Wang H, Li W, Guo R, Sun J, Cui J, Wang G, Hoffman AR and Hu JF: An intragenic long noncoding RNA interacts epigenetically with the RUNX1 promoter and enhancer chromatin DNA in hematopoietic malignancies. Int J Cancer 135: 2783-2794, 2014.

18. Choi HJ, Lui A, Ogony J, Jan R, Sims PJ and Lewis-Wambi J: Targeting interferon response genes sensitizes aromatase inhibitor resistant breast cancer cells to estrogen-induced cell death. Breast Cancer Res 17: 6, 2015.

19. Yang G, Xu Y, Chen X and Hu G: IFITM1 plays an essential role in the antiproliferative action of interferon-gamma. Oncogene 26: 594-603, 2007.

20. Khasraw M, Bell R and Dang C: Epirubicin: Is it like doxorubicin in breast cancer? A clinical review. Breast 21: 142-149, 2012.

21. Damiani RM, Moura DJ, Viau CM, Caceres RA, Henriques JA and Saffi J: Pathways of cardiac toxicity: Comparison between chemotherapeutic drugs doxorubicin and mitoxantrone. Arch Toxicol 90: 2063-2076, 2016.

22. Chelbi-Alix MK and Wietzerbin J: Interferon, a growing cytokine family: 50 years of interferon research. Biochimie 89: 713-718, 2007.

23. Parker BS, Rautela J and Hertzog PJ: Antitumour actions of interferons: Implications for cancer therapy. Nat Rev Cancer 16: 131-144, 2016.

24. Szelag M, Piaszyk-Borychowska A, Plens-Galaska M, Wesoly J and Bluyssen HA: Targeted inhibition of STATs and IRFs as a potential treatment strategy in cardiovascular disease. Oncotarget 7: 48788-48812, 2016.

25. Merches K, Khairnar V, Knuschke T, Shaabani N, Honke N, Duhan V, Recher M, Navarini AA, Hardt C, Häussinger D, et al: Virus-induced type I interferon deteriorates control of systemic pseudomonas aeruginosa infection. Cell Physiol Biochem 36: 2379-2392, 2015. 
26. Heim $\mathrm{MH}$ and Thimme R: Innate and adaptive immune responses in HCV infections. J Hepatol 61 (Suppl 1): S14-S25, 2014.

27. Zhu Y, Jia H, Chen J, Cui G, Gao H, Wei Y, Lu C, Wang L, Uede T and Diao H: Decreased osteopontin expression as a reliable prognostic indicator of improvement in pulmonary tuberculosis: impact of the level of interferon-gamma-inducible protein 10 Cell Physiol Biochem 37: 1983-1996, 2015.

28. Zhao LJ, He SF, Liu Y, Zhao P, Bian ZQ and Qi ZT: Inhibition of STAT pathway impairs anti-hepatitis $C$ virus effect of interferon alpha. Cell Physiol Biochem 40: 77-90, 2016.

29. Takaoka A and Yanai $\mathrm{H}$ : Interferon signalling network in innate defence. Cell Microbiol 8: 907-922, 2006.

30. Legrier ME, Bièche I, Gaston J, Beurdeley A, Yvonnet V, Déas O, Thuleau A, Château-Joubert S, Servely JL, Vacher S, et al: Activation of IFN/STAT1 signalling predicts response to chemotherapy in oestrogen receptor-negative breast cancer. Br J Cancer 114: 177-187, 2016.
31. Yang Y, Zhou Y, Hou J, Bai C, Li Z, Fan J, Ng IOL, Zhou W, Sun H, Dong Q, et al: Hepatic IFIT3 predicts interferon- $\alpha$ therapeutic response in patients of hepatocellular carcinoma. Hepatology: Mar 13, 2017 (Epub ahead of print).

32. Lee SC, Xu X, Lim YW, Iau P, Sukri N, Lim SE, Yap HL, Yeo WL, Tan P, Tan SH, et al: Chemotherapy-induced tumor gene expression changes in human breast cancers. Pharmacogenet Genomics 19: 181-192, 2009

33. Thomas M, Finnegan CE, Rogers KM, Purcell JW, Trimble A, Johnston PG and Boland MP: STAT1: A modulator of chemotherapy-induced apoptosis. Cancer Res 64: 8357-8364, 2004.

34. Hannesdóttir L, Tymoszuk P, Parajuli N, Wasmer MH, Philipp S, Daschil N, Datta S, Koller JB, Tripp CH, Stoitzner P, et al: Lapatinib and doxorubicin enhance the Stat1-dependent antitumor immune response. Eur J Immunol 43: 2718-2729, 2013. 